\title{
Enhancing the Chemical Flexibility of Hybrid Perovskites by Introducing Divalent Ligands
}

\author{
Paul J. Saines $^{1}$, Lydia G. Burley ${ }^{1}$, James Beecham-Lonsdale ${ }^{1}$, Anant Kumar Srivastava ${ }^{1,2}$, Ines E. Collings ${ }^{3}$ \\ ${ }^{1}$ School of Physical Sciences, University of Kent, Canterbury, Kent, United Kingdom; \\ ${ }^{2}$ Department of Materials Engineering, Indian Institute of Science (IISc), Bangalore, Karnataka, India; \\ ${ }^{3}$ Centre for X-ray Analytics, Empa - Swiss Federal Laboratories for Materials Science and Technology, Dübendorf, Switzerland;
}

\section{P.Saines@kent.ac.uk}

In recent years there has been tremendous interest in perovskite-like $\mathrm{ABX}_{3}$ hybrid frameworks, built from inorganic and organic building blocks, for their semiconducting, ferroelectric and magnetic properties. Much of the attraction in these materials lies in the well-known chemical flexibility of perovskite structures, which allows them to accommodate a wide range of cations and anions, as is well known perovskite oxides. Much of this flexibility is enhanced in inorganic-organic perovskites both with respect to their chemistry e.g. their ability to incorporate a wide range of molecular A-site cations and ligands, distortion modes and mechanical flexibility. In one key aspect, however, hybrid perovskites currently have less flexibility compared to conventional perovskites, namely the range of formal charges of cations they can incorporate. This results from the ligands in these hybrid material almost always being monovalent, which essentially restricts the A and B sites to monovalent and divalent cations, respectively.

Recent work in our group has realised a combination of monovalent and divalent ligands in perovskite-like materials via replacing $\mathrm{HCO}_{2}{ }^{-}$linker with $\mathrm{C}_{2} \mathrm{O}_{4}{ }^{2-}$ ligands. Most interestingly this has yielded $\left[\left(\mathrm{CH}_{2}\right)_{3} \mathrm{~N}\right] \mathrm{Er}\left(\mathrm{HCO}_{2}\right)_{2}\left(\mathrm{C}_{2} \mathrm{O}_{4}\right)$ and $\left[\left(\mathrm{CH}_{3}\right)_{2} \mathrm{NH}_{2}\right] \mathrm{Er}\left(\mathrm{HCO}_{2}\right)_{2}\left(\mathrm{C}_{2} \mathrm{O}_{4}\right)$, allowing monovalent organic A-site and trivalent $\mathrm{B}$-site cations to be combined for the first time in a stoichiometric $\mathrm{ABX}_{3}$ perovskite. Our presentation will discuss the synthesis, crystal structures and magnetic properties of these materials. These exhibit A-site cation ordering up to $500 \mathrm{~K}$, which will likely make related phases of interest as ferroelectrics. The greater framework flexibility in $\left[\left(\mathrm{CH}_{2}\right)_{3} \mathrm{~N}\right] \operatorname{Er}\left(\mathrm{HCO}_{2}\right)_{2}\left(\mathrm{C}_{2} \mathrm{O}_{4}\right)$ leads to it exhibiting significant anisotropic negative thermal expansion while the more rigid $\left[\left(\mathrm{CH}_{3}\right)_{2} \mathrm{NH}_{2}\right] \operatorname{Er}\left(\mathrm{HCO}_{2}\right)_{2}\left(\mathrm{C}_{2} \mathrm{O}_{4}\right)$ phase does not.

The second part of our presentation will focus on the related $\mathrm{ALn}\left(\mathrm{C}_{2} \mathrm{O}_{4}\right)_{1.5}\left(\mathrm{HCO}_{2}\right)(\mathrm{Ln}=\mathrm{Tb}-\mathrm{Er})$ phases, where we find that replacing an additional formate ligand with oxalate leads to a structure with ordered ligand vacancies. This leads to larger channels in the materials, which is likely the cause of the disorded A-site cations in these materials; ultimately the presence and nature of these A-site cations, which could not be identified crystallographically, have been confirmed by neutron and infrared spectroscopy. These two new series of materials highlight the potential to expand the flexibility of hybrid perovskite and perovskite-like materials by incorporating divalent ligands, allowing their properties to be further tailored for applications.

Keywords: pervoskites, hybrid frameworks, negative thermal expansion, spectroscopy 